\title{
DISTRIBUTED POWER ALLOCATION FOR NEXT-GENERATION COGNITIVE WIRELESS DEVICES
}

\author{
Antoni Morell, José López Vicario and Gonzalo Seco-Granados \\ Universitat Autònoma de Barcelona (UAB) \\ Telecommunications and System Engineering Department (TES) \\ e-mail: \{Antoni.Morell,Jose.Vicario,Gonzalo.Seco\}@uab.cat
}

\begin{abstract}
It is foreseen that new cognitive wireless communications devices will incorporate multiple reconfigurable radios in the near future. In this paper, we concentrate on the problem of allocating the device power to the radios and we discuss the advantages of distributed approaches in front of centralized designs. Motivated by the scalability in the design and by the potential reduction of signalling information between the transceivers and the power controller, we develop a new power allocation algorithm that is based on a novel decomposition algorithm, called coupled-decompositions method. As a result, we reduce the signalling requirements by a factor 10 with respect to centralized approaches and by a factor 3 with respect to a classical primal decomposition approach. Furthermore, the resulting technique is expected to adapt faster and more robustly to changes in the scenario conditions.
\end{abstract}

\section{INTRODUCTION}

Cognitive radio has emerged as a very promising technology to overcome the scarcity of electromagnetic spectrum by means of efficiently employing it [1]. It has been identified that only some parts of the spectrum have a high utilization factor due to the operation of current communication systems, which are referred to as the primary systems in the cognitive literature. The remaining time-frequency regions are known as the spectrum holes and the idea in cognitive radio is to allow a secondary system to exploit the available resources as far as an acceptable interference level, measured in terms of the interference temperature [1], is introduced into the primary system.

From a more practical point of view, cognitive radio is closely related to software radios. Given that a software implementation of typically hardware devices such as mixers or oscillators is possible, radio transceivers can be reconfigured so that they are no longer restricted to permanently operate

This work has been supported by the Spanish Government under project TEC2008-06305, the Catalan Government under grant 2009 SGR 298, and the Chair of Knowledge and Technology Transfer Parc de Recerca UAB Santander.

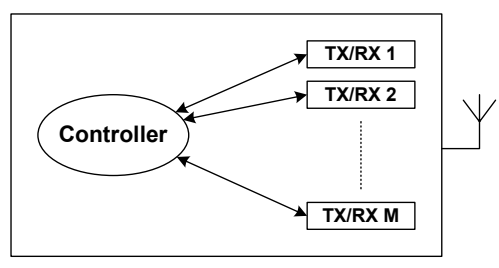

Fig. 1. Architecture of the cognitive device.

according to the same communications standard. Moreover, it is expected that next generation wireless terminals will include multiple radio transceivers in order to establish several data links, either using various channels in the same access network (e.g. IEEE 802.11n), distinct wireless systems (e.g. WIFI, WiMAX and 3G) or a combination of both [2, 3].

In this paper we consider the multiple radio scenario and we focus on the design of the communications device. Specifically, we concentrate on the allocation of the available power among the transceivers. The rest of the paper is organized as follows. Section II contains the problem formulation. Section III reviews the classical centralized solution to the posed problem and Section IV discusses alternative distributed solutions. Finally, Section V includes simulation results and Section VI concludes the paper.

\section{PROBLEM FORMULATION}

Let us assume that the total device power is $P_{T}$ and that $M$ radio transceivers are active, i.e. with the possibility to transmit. Our goal is to allocate $P_{T}$ among the $M$ radios in order to achieve the maximum possible transmission rate. Motivated by the widespread usage of Orthogonal Frequency Division Multiplexing (OFDM) and without loss of generality, we assume that the transceivers use multi-carrier modulations (or single-carrier as a particular case). Furthermore, we assume that the device has determined the maximum allowed power per subsystem and subcarrier in order to keep below the desired interference temperature. See in Figure 1 our proposed system architecture, with $M$ radios and a central controller that decides the power allocation. In the following, the terms 
radios, subsystems and transceivers are used interchangeably.

We formulate the power allocation problem as the following optimization program that maximizes the total transmission rate,

$$
\begin{array}{cc}
\max _{\left\{p_{i, j}\right\}} & \sum_{i=1}^{M} \sum_{j=1}^{N_{s}^{i}} B W_{i} \log \left(1+\frac{p_{i, j} H_{i, j}}{N_{0} B W_{i}}\right) \\
\text { s.t. } & \sum_{i=1}^{M} \sum_{j=1}^{N_{s}^{i}} p_{i, j} \leq P_{T} \\
& 0 \leq p_{i, j} \leq \bar{p}_{i, j}
\end{array}
$$

where $p_{i, j}$ is the power allocated to the $\mathrm{j}$-th subcarrier at the i-th transceiver and $\bar{p}_{i, j}$ is the maximum allowed power at the subcarrier. Note that the values $\bar{p}_{i, j}$ also fix the total power at the i-th radio to $\sum_{j=1}^{N_{s}^{i}} \bar{p}_{i, j}$, where $N_{s}^{i}$ is the number of available subcarriers. Furthermore, $B W_{i}$ stands for subcarrier bandwidth, $N_{0}$ is the noise power spectral density and $H_{i, j}$ is the channel gain at the $\mathrm{j}$-th subcarrier on the $\mathrm{i}$-th transceiver. Finally, we want to remark that a subcarrier is always managed by the same radio, being the assignment previously determined by the device.

\section{CENTRALIZED SOLUTION}

The problem in (1) can be solved by applying the Karush Kuhn Tucker (KKT) optimality conditions for convex problems [4] and the following water-filling solution is found,

$$
p_{i, j}=\left[B W_{i}\left(\frac{1}{\mu}-\frac{N_{0}}{H_{i, j}}\right)\right]_{0}^{\bar{p}_{i, j}},
$$

where

$$
[x]_{0}^{z}= \begin{cases}z & x>z \\ x & 0 \leq x \leq z \\ 0 & x<0\end{cases}
$$

and $\mu$ is such that $\sum_{i=1}^{M} \sum_{j=1}^{N_{s}^{i}} p_{i, j}=P_{T}$.

Note that the implementation of (2) in practice requires to gather all the values $H_{i, j}, \bar{p}_{i, j}$ and $B W_{i}$ at the controller, compute there the optimal allocation $\left\{p_{i, j}^{*}\right\}$ and send it back to the radios. Therefore, this option requires a large amount of signalling between the radios and the controller and to solve an optimization problem that has $N_{s}=\sum_{i=1}^{M} N_{s}^{i}$ variables. Furthermore, (2) must be recomputed whenever the parameters $H_{i, j}, \bar{p}_{i, j}$ and $B W_{i}$ change or when a radio is reconfigured. In order to overcome these impracticalities, we explore alternative solutions in the next section.

\section{DISTRIBUTED SOLUTION}

A different approach to the problem is to use the controller to decide only the amount of transmission power that is given to each transceiver and let the radios to internally allocate it, resulting in a scalable solution. Our motivation is to reduce the amount of signalling and to spread the processing load inside the receiver by means of parallel computing. Note that transmitting only the power per subsystem instead of the power per subcarrier simplifies the system interfaces, makes the communication between the radios and the controller easier and may help in future standards.

Assuming that each radio is able to compute its optimal power allocation, we can reformulate the problem in (1) as

$$
\begin{aligned}
& \max _{\left\{P_{i}\right\}} \quad \sum_{i=1}^{M} r_{i}\left(P_{i}\right) \\
& \text { s.t. } \quad \sum_{i=1}^{M} P_{i} \leq P_{T}
\end{aligned}
$$

where $r_{i}\left(P_{i}\right)$ represents the internal power allocation problem, expressed as

$$
r_{i}\left(P_{i}\right)=\left\{\begin{array}{cc}
\max _{\left\{p_{i, j}\right\}} & \sum_{j=1}^{N_{s}^{i}} B W_{i} \log \left(1+\frac{p_{i, j} H_{i, j}}{N_{0} B W_{i}}\right) \\
\text { s.t. } & \sum_{j=1}^{N_{s}^{i}} p_{i, j} \leq P_{i} \\
& 0 \leq p_{i, j} \leq \bar{p}_{i, j}
\end{array}\right.
$$

Using this alternative formulation, we want to establish a dialogue between the radios and the transceivers in order to find the optimal power allocation. This dialogue should contain only aggregated values such as the $P_{i}$ 's and avoid to signal specific subcarrier powers and parameters, which remain as internal variables of the radios.

\subsection{Primal Decomposition Approach}

A classical primal decomposition approach [5, Sec. 6.4.2] can be applied to solve (4). In this way, the vector $\boldsymbol{P}=$ $\left[P_{1}, \ldots, P_{M}\right]^{T}$ that collects the transmission powers of the transceivers is updated using the following rule,

$$
\boldsymbol{P}^{k+1}=\left[\boldsymbol{P}^{k}+\alpha^{k} \boldsymbol{s}^{k}\right]^{\dagger}
$$

where $k$ indexes iterations, $\alpha^{k}$ is the step-size of the method and $[\boldsymbol{P}]^{\dagger}$ is the projection of $\boldsymbol{P}$ on the subspace determined by $\sum_{i=1}^{M} P_{i} \leq P_{T}, P_{i} \geq 0$ and $P_{i} \leq \sum_{j} \bar{p}_{i, j}, \forall i$. Finally, $\boldsymbol{s}^{k}$ is the subgradient of $\sum_{i=1}^{M} r_{i}\left(P_{i}\right)$ at $\boldsymbol{P}^{k}$. Once the problems in (5) are solved (internally at the radios), $s^{k}$ is readily expressed as

$$
\boldsymbol{s}^{k}=-\left[\lambda_{1}^{*}\left(P_{1}^{k}\right), \ldots, \lambda_{M}^{*}\left(P_{M}^{k}\right)\right]^{T},
$$

where $\lambda_{i}^{*}\left(P_{i}^{k}\right)$ is the optimum value of the Lagrange multiplier associated to the constraint $\sum_{j=1}^{N_{s}^{i}} p_{i, j} \leq P_{i}^{k}$ in (5). Therefore, the radios exchange only the transmission powers in $\boldsymbol{P}^{k}$ and the subgradients in $\boldsymbol{s}^{k}$ using this approach.

However, the method experiences a low speed of convergence and furthermore, as far as the step-size has to be manually adjusted (see [6]), there is a very low probability of selecting the optimal step at each time instant. For the sake of brevity, we do not include here another wide-used decomposition approach, that is, dual decomposition [5, Sec. 6.4.1], but we evaluate it in our results. In the following, we derive the solution of (4) using a new method developed by the authors $[7,8]$. It outperforms primal and dual decompositions in terms of convergence speed and furthermore, it requires no parameter adjustment. 


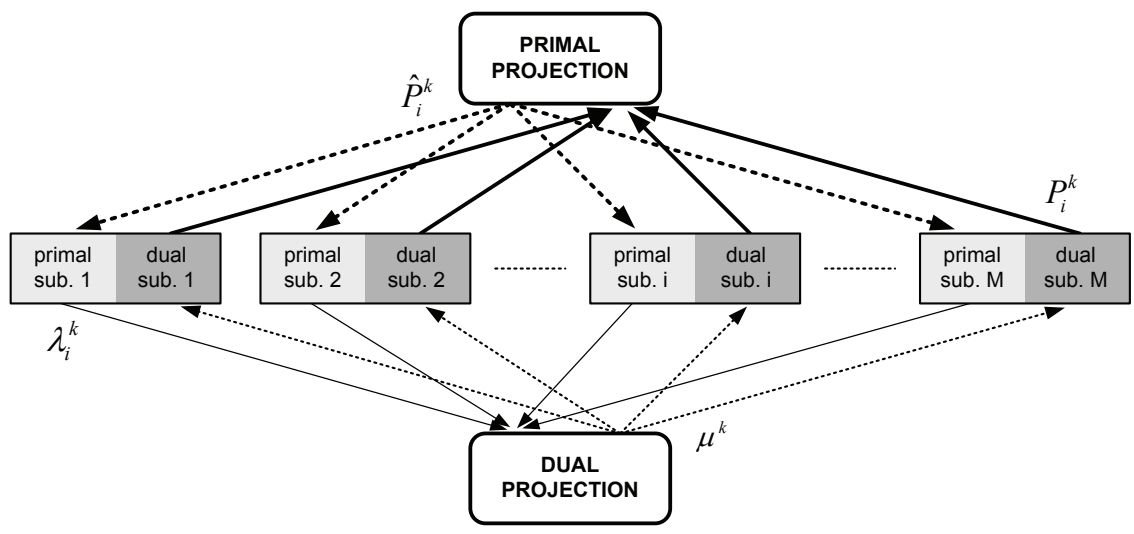

Fig. 2. Block diagram of the coupled-decompositions method.

\subsection{Coupled-Decompositions Approach}

The coupled-decompositions combines the ideas from primal and dual decompositions in a single technique. It is important to remark that this solution is different to other approaches in the literature that use primal and dual solution domains simultaneously. For example, [6] shows how to serially concatenate an arbitrary number of primal and dual decompositions; and the Mean Value Cross (MVC) decomposition method [9] or the primal-dual interior point methods [4, Sec. 11.7] use both solution domains but do not exploit the problem separability, as opposite to classical decomposition techniques.

The coupled-decompositions method (CDM) can be summarized in four steps, namely: i) dual subproblems, ii) primal projection, iii) primal subproblems and iv) dual projection. This is also the execution order (see Figure 2) that iteratively finds the optimal water-level $\mu$. The definition of the subproblems in the CDM coincides with the primal and dual subproblems that appear in classical decompositions whereas the projections are completely redefined $[7,8]$.

In the following, we detail the steps of the method for the proposed problem. Given $\mu^{k}$ at iteration $k$, we compute the following dual subproblems:

$$
\begin{array}{cc}
\max _{\left\{p_{i, j}\right\}, P_{i}^{k}} & \sum_{j=1}^{N_{s}^{i}} B W_{i} \log \left(1+\frac{p_{i, j} H_{i, j}}{N_{0} B W_{i}}\right)-\mu^{k} P_{i}^{k} \\
\text { s.t. } & \sum_{j=1}^{N_{s}^{i}} p_{i, j} \leq P_{i}^{k} \\
& 0 \leq p_{i, j} \leq \bar{p}_{i, j}
\end{array}
$$

Applying the KKT optimality conditions, the closed-form solution of (8) is

$$
\begin{gathered}
p_{i, j}=\left[B W_{i}\left(\frac{1}{\mu^{k}}-\frac{N_{0}}{H_{i, j}}\right)\right]_{0}^{\bar{p}_{i, j}} . \\
P_{i}^{k}=\sum_{j=1}^{N_{s}^{i}} p_{i, j}
\end{gathered}
$$

After the dual subproblems, the values in $\boldsymbol{P}^{k}$ may not attain the total power constraint. The primal projection obtains then the corrected values in $\hat{\boldsymbol{P}}^{k}$ by computing the projection on the hyperplane defined by $\sum_{i=1}^{M} P_{i}=P_{T}$ and the feasible range of each $P_{i}$, that is,

$$
\begin{array}{cc}
\min _{\left\{\hat{P}_{i}^{k}\right\}} & \left\|\hat{\boldsymbol{P}}^{k}-\boldsymbol{P}^{k}\right\|^{2} \\
\text { s.t. } & \sum_{i=1}^{M} \hat{P}_{i}^{k}=P_{T} \\
& 0 \leq \hat{\hat{P}}_{i}^{k} \leq \sum_{j} \bar{p}_{i, j}, \quad \forall i
\end{array}
$$

The corrected values $\hat{P}_{i}^{k}$ are then used in the primal subproblems, already defined in (5). These problems are internally solved at each radio transceiver using standard waterfilling techniques such as the bisection method. As a result of the primal subproblems, the Lagrange multipliers associated to the constraints $\sum_{j=1}^{N_{s}^{i}} p_{i, j} \leq \hat{P}_{i}^{k}$ at iteration $k$, i.e. $\lambda_{i}^{k}$, are obtained. Note that if $\hat{P}_{i}^{k}<0$, the corresponding primal subproblem has no feasible solution and therefore, it returns no value of $\lambda_{i}^{k}$.

Finally, the dual projection updates $\mu^{k}$ to $\mu^{k+1}$ using the list $\left\{\lambda_{i}^{k}\right\}$. It is straightforward to update the water-level $\mu$ in our problem by computing

$$
\mu^{k+1}=\min \left\{\lambda_{i}^{k}\right\} .
$$

The interested reader can find more details about the dual projection and the CDM as well as its proof in $[7,8]$. In the following, we provide a brief interpretation of the technique from a resource-price perspective, which is a common way to interpret primal variables (resources) and dual variables (prices to be paid) in convex problems.

\section{Resource-price interpretation}

The method starts with an initial price $\mu^{0}$. First, the radios buy a certain amount of transmission power taking into account the current price (dual subproblems). Thereafter, the controller modifies the previous purchases in order to limit the total amount of power to $P_{T}$ (primal projection). However, since the correction has not taken into account the unavoidable price modification, the radios check for the price they would pay for the new allocation (primal subproblems). If the new prices do not correspond to the allocated resources, the controller sorts out a new price $\mu^{k+1}$ (dual projection). The process is repeated until a consensus price is found. 
Table 1. Description of the subsystems

\begin{tabular}{cccc}
$\begin{array}{c}\text { Subsystem } \\
\text { number }\end{array}$ & $\begin{array}{c}\text { Number of } \\
\text { subcarriers }\end{array}$ & $\begin{array}{c}\text { Subcarrier } \\
\text { bandwidth }\left(B W_{i}\right)\end{array}$ & $\begin{array}{c}\text { Subsystem } \\
\text { bandwidth }\end{array}$ \\
\hline \hline 1 & 256 & $6 \mathrm{kHz}$ & $1.536 \mathrm{MHz}$ \\
\hline 2 & 256 & $12 \mathrm{kHz}$ & $3.072 \mathrm{MHz}$ \\
\hline 3 & 128 & $10 \mathrm{kHz}$ & $1.28 \mathrm{MHz}$ \\
\hline
\end{tabular}

Note that using the CDM, we exchange the transmission powers $\left\{P_{i}^{k}\right\}$ and $\left\{\hat{P}_{i}^{k}\right\}$, the validation prices $\left\{\lambda_{i}^{k}\right\}$ and the consensus price $\mu^{k+1}$.

\section{Summary in algorithmic form}

PROPOSED POWER ALLOCATION ALGORITHM

Take starting point $\mu^{0}=0$ and let $k=0$.

Repeat

1. Compute the primal variables $P_{i}^{k}$ using $\mu^{k}$ in the dual subproblems (9).

2. Apply the primal projection to get the values $\hat{P}_{i}^{k}$ from $P_{i}^{k}$ as in (10).

3. Use the primal subproblems in (5) and get $\lambda_{i}^{k}$ from $\hat{P}_{i}^{k}$.

4. Update $\mu^{k}$ using the dual projection in (11), i.e. $\mu^{k+1}=\min \left\{\lambda_{i}^{k+1}\right\}$.

Until convergence.

Finally, note that when a distributed solution is employed, the radio transceivers become transparent to the controller, that is, the algorithm is not affected by changes on the configuration of the radios or, in other words, it is able to more easily adapt to channel fluctuations or reconfigurations on the radios. If we use a centralized approach instead, any change requires to reset the algorithm.

\section{SIMULATION RESULTS}

Let us assume a communication device that has three radio transceivers. The radios use mutli-carrier modulations and their configuration parameters are summarized in Table 1 . We specify the number of available subcarriers, the subcarrier bandwidth $B W_{i}$ and the total spanned bandwidth at each subsystem. Overall, the device manages $5.888 \mathrm{MHz}$ and $N_{s}=$ 640 subcarriers. We assume that the channels in the three subsystems are frequency selective and that each channel has a length of 20 taps as well as an exponential power delay profile with $1 \mathrm{~ms}$ of delay spread. Mean channel gain is $0 \mathrm{~dB}$ in transceiver $1,-10 \mathrm{~dB}$ in transceiver 2 and $-5 \mathrm{~dB}$ in transceiver 3 . We fix the noise power at the first radio to $\sigma_{n}^{2}$ and the total available power to $P_{T}(d B)=\sigma_{n}^{2}(d B)+10 \log _{10}(640)+5$.

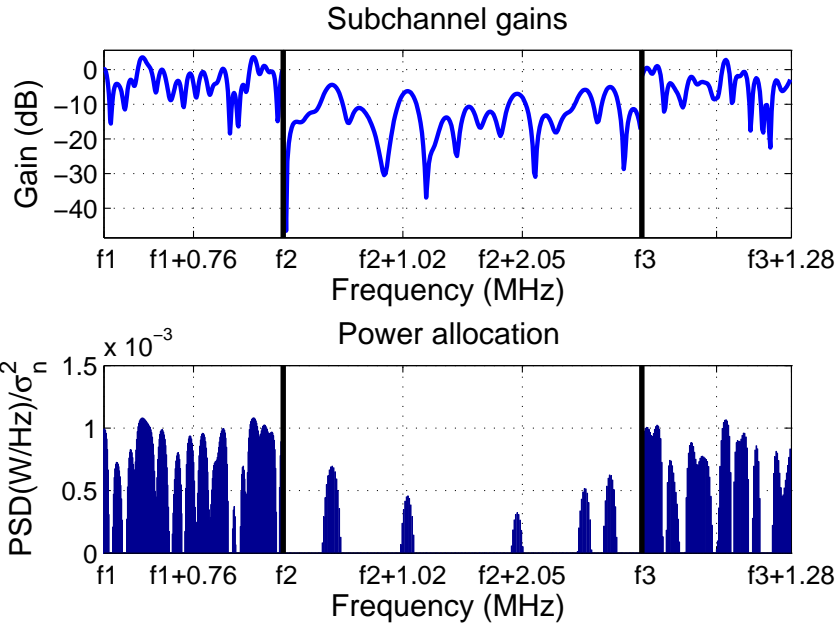

Fig. 3. Distributed water-filling example. Top: channel gains. Bottom: power allocation. In $f 1-f 3$ we have the initial band frequencies of subsystems 1-3.

Moreover, we assume that the noise power spectral density (PSD) is flat over frequency and therefore, $N_{0}=\sigma_{n}^{2} / B W_{1}$. Finally, we consider in our example that no further restriction is imposed to the power, which implies $\bar{p}_{i, j}=\infty, \forall i, j$

In Figure 3, we plot a channel realization for each subsystem (top) and the resulting power allocation (bottom). We confirm that most of the power is allocated to transceivers 1 and 3 , which are the ones that have the best channel condition. On the contrary, subsystem 2 only allocates power to a few subcarriers with the highest channel gains. Notwithstanding, in absolute terms, transceiver 2 receives a relatively large allocation in order to exploit the higher subcarrier bandwidth.

In Figure 4 we show the evolution of the Normalized Mean Squared Error (NMSE) in the power allocation with respect to the number of messages exchanged between the radios. We define the NMSE as

$$
N M S E=\frac{\sum_{i, j}\left(p_{i, j}-p_{i, j}^{*}\right)^{2}}{\sum_{i, j}\left(p_{i, j}^{*}\right)^{2}},
$$

where $p_{i, j}^{*}$ is the optimal value of $p_{i, j}$. Note that this metric has more interest than others, such as the computational time or the speed of convergence of the methods, in the problem we are dealing with. Since signalling is in general more restrictive than processing in terms of latency, this measure allows us to establish a fair comparison between methods. In particular, we evaluate here the coupled-decompositions method, a primal decomposition strategy, a dual decomposition solution and the bisection method (as an example of a centralized approach). Note that the proposed technique exchanges $4 \cdot M$ messages at each iteration, whereas a primal or a dual decomposition strategy needs $2 \cdot M$ messages per iteration.

Results show that in case of employing the proposed CDM, around 150 messages suffice in order to achieve the optimal solution (with a NMSE $=10^{-10}$ ). Significant extra messag- 


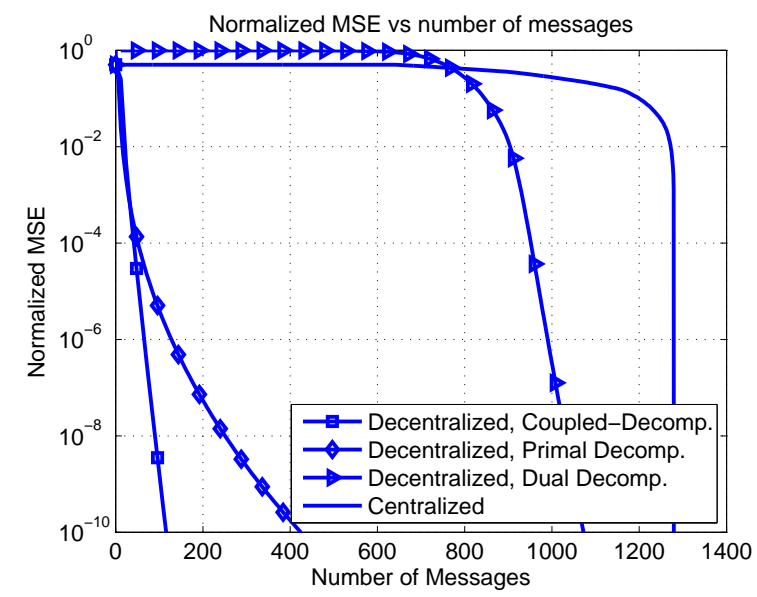

Fig. 4. NMSE vs number of signaling messages.

ing (by a factor 3 ) is required in primal decomposition when a step size $\alpha(k)=1 / \sqrt{k}$ is used in the projected subgradient approach and it increases when we use a dual decomposition keeping the same step-size. In both cases, we have manually adjusted it in order to achieve good performance in terms of convergence speed, so in practice the CDM would have even a greater advantage with respect to classical decompositions.

Finally, a centralized approach requires to exchange 1280 messages, i.e. $2 \cdot N_{s}$. In this case, we first signal all the channel gains to the controller and therefore, we need to transmit 640 messages in this first phase. Note that we do not consider here the signalling of the values in $\bar{p}_{i, j}$, and therefore, the results of the centralized approach are optimistic. In a second phase, the optimal channel allocation is sent back to the radios and we update the NMSE every time a new subcarrier allocation is received. Note in the figure that this solution obtains a good performance only at the last message exchanges because the error that a reduced number of not properly allocated subcarriers introduces is relatively high. Note also that in practice we would need to receive the complete allocation to ensure that the power constraint is accomplished. This problem is circumvented in our solution because the total power constraint is enforced at each iteration.

\section{CONCLUSIONS}

In this paper, we have discussed and analyzed the alternative power allocation solutions that can be implemented in future multiple-radio cognitive devices. We have shown that a distributed approach to the problem is advantageous in front of a classical centralized strategy because: i) signalling is reduced, ii) the design is scalable and iii) the resulting algorithm adapts faster to changes in the scenario or in the transceivers. Moreover, three distributed optimization mechanisms have been evaluated: the classical primal and dual decomposition approaches and the author's coupled-decompositions method.

The number of exchanged messages between the radios and the controller has been used as a performance metric. Assuming that signalling is more restrictive than processing in terms of latency, this quantity is more useful than, for example, the convergence speed of the methods. Results have shown that, in order to guarantee a small allocation error, centralized techniques need to signal all the parameters to the controller and retrieve essentially the complete allocation afterwards. On the contrary, distributed strategies achieve a good performance with a much lower number of messages. Comparing distributed methods, we realize that the proposed coupled-decompositions method outperforms a classical primal decomposition by a factor of 3 in our simulations. Moreover, it is expected that the proposed solution is more robust against changes in the scenario because it requires no adjustment, but this remains as future work.

\section{REFERENCES}

[1] S. Haykin, "Cognitive Radio: Brain-Empowered Wireless Communications," IEEE Journal on Selected Areas in Communications, Vol. 23, No. 2, Feb 2005.

[2] I.F. Akyildiz, S. Mohanty, and Jiang Xie, "A Ubiquitous Mobile Communication Architecture for NextGeneration Heterogeneous Wireless Systems," IEEE Communications Magazine, Vol. 43, No. 6, pp. S29-S36, Jun 2005 .

[3] D. Wang, K. Miao, V. John, S. Rungta, and W. Chan, "Considering Wireless Mesh Network with Heterogeneous Multiple Radios," IEEE WiCom, pp. 1681-1684, Sep 2007.

[4] L. Boyd and S. Vandenberghe, Convex optimization, Cambridge University Press, 2003.

[5] D.P. Bertsekas, Nonlinear Programming, Belmont, MA, USA: Athena Scientific, 1999.

[6] D.P. Palomar and M. Chiang, "Alternative Distributed Algorithms for Network Utility Maximization: Framework and Applications," in IEEE Tran. on Automatic Control, Vol. 52, No. 12, pp. 2254-2269, Dec 2007.

[7] A. Morell, A Convex Decomposition Perspective on Dynamic Bandwidth Allocation and Applications, Ph.D. Dissertation available at http://spcomnav.uab.es, 2008.

[8] A. Morell, G. Seco-Granados, and J.L. Vicario, "Fair Adaptive Bandwidth and Subchannel Allocation in the WiMAX Uplink," EURASIP Journal on Wireless Communications and Networking, Vol. 2009, Article ID 918261, 13 pages, doi:10.1155/2009/918261, 2009.

[9] K. Holmberg and K.C. Kiwiel, "Mean Value Cross Decomposition for Nonlinear Convex Problems," Optimization Methods and Software, Vol. 21, No. 3, pp. 401-417, Jun 2006. 